\title{
Post-trial Access in Maternal Vaccine Trials
}

\author{
I. M. A. A. Van Roessel, BSc ${ }^{1}$ \\ N. I. Mazur, MD, MSc ${ }^{1,2,3}$ \\ S. K. Shah, $\mathrm{PhD}^{2,3}$ \\ L. Bont, MD, $\mathrm{PhD}^{1}$ \\ R. Van Der Graaf, PhD ${ }^{4}$
}

${ }^{1}$ Division of Paediatric Immunology and Infectious Diseases, University Medical Centre Utrecht, Utrecht, The Netherlands

Address for correspondence L. Bont, MD, PhD, Division of Paediatric

2 Lurie Children's Hospital, Smith Child Health Research and Advocacy Immunology and Infectious Diseases, University Medical Centre Center, Chicago, United States

${ }^{3}$ Department of Pediatrics, Northwestern University Medical School, Chicago, United States

${ }^{4}$ Julius Center for Health Sciences and Primary Care, University

Medical Centre Utrecht, Utrecht, The Netherlands

Am J Perinatol 2019;36(suppl S2):S41-S47.

\begin{abstract}
Keywords

- post-trial access

- maternal vaccination

- vaccine trials

- research

- ethical

Provisions for post-trial access (PTA) of the experimental intervention are required before the start of a clinical trial. Although there has been ample attention for PTA in the context of preventive vaccine research, discussions on PTA barely include maternal vaccine trials in which mother-infant pairs are exposed to the intervention. In maternal vaccination trials, specific PTA arrangements are required because pregnancy is transient and PTA may apply to the next pregnancy or the child. In this article, we examine the application and adherence to PTA in the context of maternal vaccine trials. We focused on differences between publications before and after 2000 when international ethical guidance documents formalized PTA requirements. Randomized maternal vaccine trials were included after a systematic search for clinical trials in phases II and III with a maternal vaccine as intervention. We used PTA as defined at the time of publication in the World Medical Association's Declaration of Helsinki (DoH) or in the ethical guidelines of the Council for International Organizations of Medical Sciences (CIOMS). In addition, we investigated whether PTA was included in the trial design. Therefore, we contacted principal investigators (PI's) of the publications found in the review to fill out a questionnaire regarding provisions for PTA. Before and after 2000, no trial articles examined in the systematic review described PTA in their trial publication (0/7, $0 \%$ and $0 / 17,0 \%$, respectively). In addition, more than half of the Pl's of the trials found were not familiar with PTA recommendations in international ethical guidelines. Most cases of PTA included making knowledge available by publishing the results of the trial. The revision of the $\mathrm{DoH}$ in 2002 and the CIOMS ethical guidelines in 2002 has not resulted in increased PTA provisions for maternal vaccination trials. PTA is a shared responsibility of various stakeholders including sponsors, Institutional Review Boards, regulators, political entities, and researchers. Inclusion of PTA provisions in trial protocols and publications on maternal vaccination trials is essential to increase transparency on the form and content of these provisions.
\end{abstract}

Maternal vaccination is an intervention to protect newborns from life-threatening infectious disease in the first month of life. Maternal immunization can protect newborns via an immunoglobulin $G(\operatorname{Ig} G$ ) antibody response to an (in)acti- vated micro-organism. IgG antibodies are transported actively across the placenta to the fetus and thereby provide passive immunity in the newborn which lasts for the first 6 months of life. After this period, the immune system of the
Copyright (c) 2019 by Thieme Medical Publishers, Inc., 333 Seventh Avenue, New York, NY 10001, USA. Tel: +1(212) 584-4662.
DOI https://doi.org/ 10.1055/s-0039-1691799. ISSN 0735-1631. 
child can generate active antibody responses via infant vaccination. ${ }^{1}$ Maternal vaccines for several pathogens are already approved and recommended for pregnant women in various countries: influenza, tetanus, and pertussis, while meningo-/pneumococcus, group B Streptococcus, and Haemophilus influenzae type B are still in clinical development and not yet recommended. More vaccines are in the pipeline: cytomegalovirus, herpes simplex virus, and respiratory syncytial virus (RSV). ${ }^{2,3}$ Despite maternal vaccination as a rapidly growing field, there is still hesitancy to vaccinate pregnant women. ${ }^{4}$ However, various RSV trials are now moving forward from early to late phase clinical trials. ${ }^{5}$ This development requires reflection on post-trial access (PTA) provisions.

International ethical guidelines for research involving human subjects support the value of PTA requirement for clinical trials. In 2000, PTA was added to the Declaration of Helsinki (DoH) paragraph 20. The DoH stated that "At the conclusion of the study, every patient entered into the study should be assured of access to the best proven prophylactic, diagnostic and therapeutic methods identified by the study." Various other international ethical frameworks, including the National Bioethics Advisory Commission, the Nuffield Council on Bioethics, and the Council for International Organizations of Medical Sciences (CIOMS) have adopted PTA requirements in their guidance documents (- Fig. 1) ${ }^{6-9}$ Despite agreement about the importance of the requirement, there is extensive discussion about the underlying rationales for PTA, about its content, the length, and to whom it applies. In general, according to small interpretations fulfilling the PTA requirement implies making provisions for continued access to interventions identified as beneficial, but broader interpretations also include provisions for transitioning participants who continue to need care or preventive measures to appropriate health services when the study has ended. The responsibility to fulfill PTA requirements is typically shared among several stakeholders including sponsors, regulators, political entities, and researchers. The shared responsibility makes providing PTA a complex issue. Investigators of a study cannot provide PTA alone and are dependent on the government, pharmaceuticals, and sponsors. ${ }^{10}$

Although there is ample literature on PTA in the context of human immunodeficiency virus (HIV) prevention research, scholars have barely reported on PTA for maternal vaccination studies. Poor attention is remarkable since it is reasonable to assume that in the case of maternal vaccination, PTA could be conceived as access to the vaccine in future pregnancies not only for women receiving placebo.

To understand why PTA requirements receive limited attention in discussions about maternal vaccination studies, we performed an in-depth study whether and how PTA requirements as formulated in the CIOMS guidelines and the $\mathrm{DoH}$ are included in publications on late phase maternal vaccine trials and contacted principle investigators of these publications about provisions made. We compared PTA provisions made before and after 2000 , when the guidelines were not in place yet. Furthermore, this study identifies best practices for implementation of PTA provisions.

\section{Materials and Methods}

\section{Systematic Review}

Randomized maternal vaccine trials were included after a systematic search (- Supplementary Appendix A, available in the online version) in PubMed for clinical trials in phases II and III with a maternal vaccine or prophylaxis as intervention. All articles were screened for eligibility by two people independently, using Rayyan. ${ }^{11}$ The World Health Organization (WHO) clinical trial registry and ClinicalTrials. gov were searched for phase II/III maternal vaccine trials, using the same in- and exclusion criteria as for PubMed. Relevant completed or ongoing trials were included, and withdrawn trials were excluded. Trials with no article available were also excluded for the systematic review (-Table 1). Trials before 2000 and after 2000 were compared since PTA was first included in the ethical guidelines in 2000.

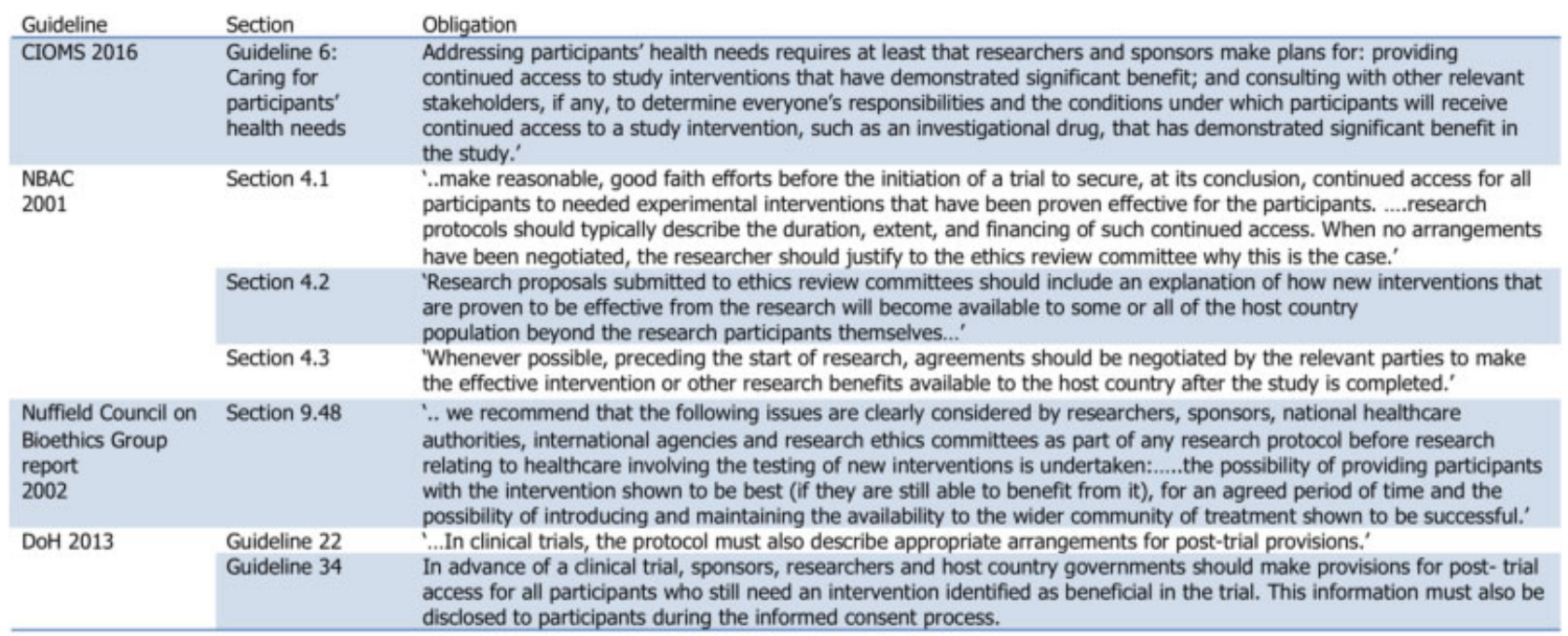

Fig. 1 Overview posttrial access in various ethical guidelines. ${ }^{8-11}$ 
Table 1 In- and exclusion criteria systematic review

\begin{tabular}{|c|c|}
\hline Inclusion & Exclusion \\
\hline \multirow[t]{2}{*}{ Pregnant women } & $\begin{array}{l}\text { Women of childbearing age/ } \\
\text { nonpregnant women }\end{array}$ \\
\hline & Animals \\
\hline Passive or active immunization & No vaccination \\
\hline \multirow[t]{2}{*}{ Maternal vaccine trials } & $\begin{array}{l}\text { HPV } 16 / 18 \text { trials } \\
\text { (because goal of vaccination } \\
\text { is not child protection) }\end{array}$ \\
\hline & HIV PMTCT trials \\
\hline Phases I/II, II, and III trials & Phases I and IV trials \\
\hline \multicolumn{2}{|l|}{$\begin{array}{l}\text { Positive and negative } \\
\text { outcomes }\end{array}$} \\
\hline $\begin{array}{l}\text { Prospective randomized } \\
\text { controlled trials }\end{array}$ & Editorial \\
\hline \multirow{5}{*}{$\begin{array}{l}\text { Secondary analysis (NB: if } \\
\text { duplicate, only primary } \\
\text { article was included) }\end{array}$} & Review \\
\hline & No PDF available \\
\hline & $\begin{array}{l}\text { No author information } \\
\text { provided }\end{array}$ \\
\hline & Language barrier \\
\hline & $\begin{array}{l}\text { Duplicates or secondary } \\
\text { analysis while primary article } \\
\text { was already included }\end{array}$ \\
\hline
\end{tabular}

Abbreviations: HIV, human immunodeficiency virus; HPV, human papillomavirus; PMTCT, prevention of mother-to-child transmission.

\section{Definitions}

A vaccine was defined according to the WHO's definition: an intervention that augments immunity to a particular disease, which contains an agent that resembles a diseasecausing microorganism. ${ }^{12}$ Human intravenous immunoglobulin (HIVIG) and intravenous immunoglobulin (IVIG) trials were included as relevant interventions. HIV prevention of mother-to-child transmission trials, HBV tenofovir trials, and antibiotic prophylaxis were not included since they do not enhance immunity or contain a part of a microbe, but only prevent mother-to-child transmission by reducing the viral load. Full-text articles were screened for description of PTA as defined at the time of publication in the DoH or CIOMS (-Fig. 1).

\section{Data Collection}

The principal investigator (PI) of each trial was contacted and asked to fill out a short questionnaire regarding provisions for PTA in the trial design. Contact with the PI was still attempted for trials that were excluded from the systematic review if there was no article available. All ongoing and completed trials from the WHO clinical trial registry and ClinicalTrials.gov were included. PI's were contacted first by telephone, then e-mail for a maximum of three times of follow-up. They were also asked to inform us if they were not willing to participate. The questionnaire was shared using Qualtrics software, version 2017. Where questionnaire data are factual, facts were verified against other sources such as trial protocols. The methods were modeled after the methodology of Haire and Jordens, Developing World Bioethics, 2015 in which PI's of phase IIB/III HIV efficacy trials were contacted in an empirical study of PTA. ${ }^{12}$

\section{Results}

\section{Systematic Review}

Twenty-four maternal vaccine trials were identified for this systematic review (-Fig. 2, - Table 2). Before and after 2000, no trial articles examined in this systematic review described PTA in their trial report $(0 / 7,0 \%$ and $0 / 17,0 \%$, respectively); $6 / 17$ (35.3\%) trials mentioned that they were conducted in accordance with the DoH in their trial report but did not specify PTA provisions.

\section{Questionnaires}

Thirty trials were identified as the PI was contacted to collect data on PTA (-Tables 2 and 3). Thirty trials were eligible for the qualitative analyses. Out of 30 PI's, 17 responded to the questionnaire. One PI was not willing to participate, and 12 investigators did not respond after follow-up. Eighty-two per cent (14/17) of PI's from trials conducted after 2000 described provisions regarding PTA.

\section{Awareness}

The majority $(59 \%, 10 / 17)$ of the Pl's for maternal vaccine trials were not aware of post-trial recommendations in international ethical guidelines. In several cases, the PI was not aware of PTA, but the PI indicated that he or she had made provisions for PTA. Half of the PI's who were aware of posttrial provisions still did not describe them for their trial.

\section{Best Practice}

From the PTA provisions that have been made by investigators in phase II/III maternal vaccine trials, most of them included making knowledge available for the population and transition to care when the research is concluded (79\% [11/14] and 64\% [9/14]). Researchers who described PTA provisions shared their protocol. Some only described making knowledge available through publication of the article as PTA provision in their protocol. Researchers indicated that the best way to incorporate obligation of PTA in the future would be to state intentions to local Institutional Review Board and Research Ethics Committee. Several PI's indicated incorporating obligations in trial protocols and informed consent to be a best practice to conform to PTA obligations.

\section{Challenges}

Researchers reported different reasons to not address PTA. One reason was that PTA was felt to be the responsibility of the local government rather than that of the researcher. Other challenges included the lack of proven benefit, awaiting WHO recommendation or national approval, a delay caused by lack of funding, consulting with other relevant stakeholders, and determining the responsibilities of different stakeholders. Finally, the PI's indicated that there was no 


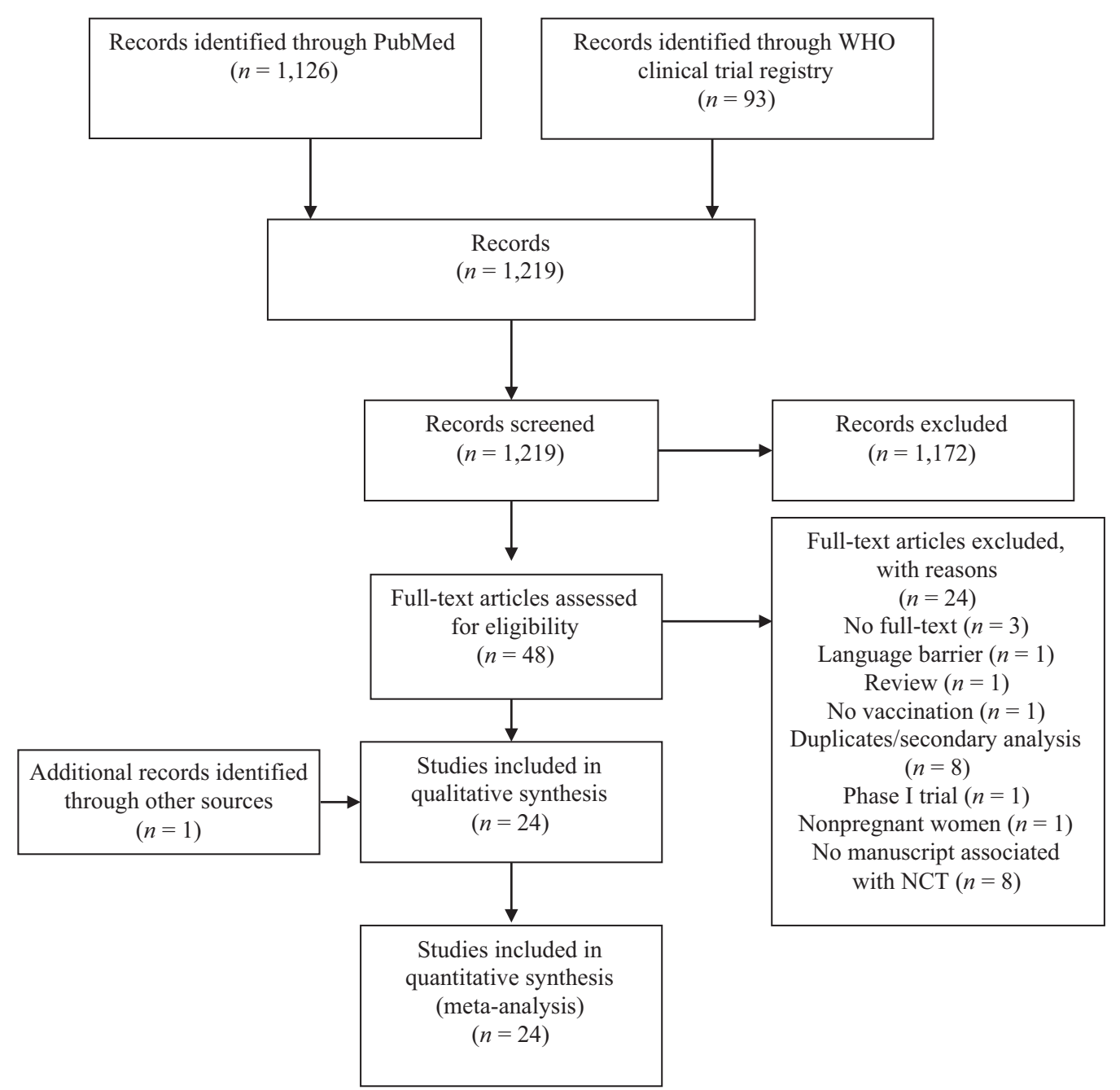

Fig. 2 Flow chart systematic review maternal vaccine trials. ${ }^{13}$

practical guidance available, or at least none that the investigators were aware of.

\section{Discussion}

No trial article, before and after 2000, examined in this systematic review described specifically PTA in their publication. However, $82 \%$ of the trials after 2000 described provisions regarding PTA elsewhere, for example, in trial protocols. Most of them included making knowledge available for the population. The percentage of $82 \%$ may be relatively high but should be critically examined. Several researchers published the article and did not address other aspects of PTA that are important for the community, such as providing continued access for study participants and making the vaccine available for the population.

The majority of the PI's was not aware of the concept of PTA. Remarkably, there were several cases in which the PI was not aware of PTA, but still indicated that he or she had made provisions for PTA. Lack of awareness of the concept PTA despite inclusion of provisions may indicate that provisions were included in the research without knowledge of the underlying concept of PTA such as publication of results without awareness of PTA obligations. Half of the PI's who were aware of posttrial provisions still did not describe them for their trial. This finding demonstrates a gap in implementation of PTA guidelines and not only awareness.

Best practices and obstacles in the process of PTA were identified. According to researchers, the best way to incorporate PTA obligations for trial planning in the future would be including PTA provisions in the protocol submitted to the ethical committees. Obstacles to including PTA in the trial planning were shared responsibilities, lack of funding, and awaiting proven benefit and recommendation. Lack of practical guidance available for PTA provisions in prevention trials remains an important obstacle and the creation of such guidance may also enhance awareness.

This study provides the first data on whether researchers implement provisions in the planning of published maternal vaccine trials. Thorough methodology was used including a systematic search with an extensive search term and careful examination of trials by two independent researchers. Furthermore, PI's of the trial were contacted to verify whether PTA provisions were included in the trial planning process. Where possible, facts have been verified against other sources, such as trial protocols. A limitation of this 
Post-trial Access in Maternal Vaccine Trials Van Roessel et al. \$45

Table 2 Maternal vaccine trials overview systematic review

\begin{tabular}{|c|c|c|c|c|c|}
\hline & Pathogen & Article & Year & Country & Study size \\
\hline \multirow[t]{7}{*}{ Before 2000} & \multirow[t]{2}{*}{ Pneumococcal } & Quiambao et a ${ }^{14}$ & 1994-1995 & Philippines & 160 \\
\hline & & Munoz et $\mathrm{al}^{15}$ & 1995-1996 & United States & 60 \\
\hline & Meningococcal & Shahid et al ${ }^{16}$ & 1995-1998 & Bangladesh & 157 \\
\hline & Hib & Mulholland et a ${ }^{17}$ & 1993-1995 & The Gambia & 451 \\
\hline & Tetanus & Newell et al ${ }^{18}$ & $1961-1966$ & Colombia & 1,618 \\
\hline & Varicella zoster & Koren et $\mathrm{al}^{19}$ & $1999-2000$ & United States & 60 \\
\hline & RSV & Munoz et al ${ }^{20}$ & 1999-2002 & United States & 35 \\
\hline \multirow[t]{19}{*}{ After $2000^{\mathrm{b}}$} & \multirow[t]{9}{*}{ Influenza } & Jackson et $\mathrm{al}^{21}$ & 2009 & United States & 120 \\
\hline & & Tielsch et al ${ }^{22}$ & $2010-2018$ & Nepal & $3,000^{\mathrm{a}}$ \\
\hline & & \multirow[t]{3}{*}{ Omer et al ${ }^{23}$} & $2011-2013$ & Nepal & 3,700 \\
\hline & & & $2011-2013$ & Mali & 4,193 \\
\hline & & & 2012 & South Africa & 2,108 \\
\hline & & Tsatsaris et al ${ }^{24}$ & 2009 & France & 107 \\
\hline & & Abzug et $\mathrm{al}^{25}$ & 2009 & United States & 127 \\
\hline & & Madhi et $\mathrm{al}^{26}$ & $2011-2012$ & South Africa & 2,310 \\
\hline & & Zaman et al ${ }^{27}$ & $2004-2005$ & Bangladesh & 340 \\
\hline & Tetanus & Salama et $a^{28}$ & $2002-2003$ & Egypt & 131 \\
\hline & \multirow[t]{3}{*}{ GBS } & Donders et $\mathrm{al}^{29}$ & $2011-2013$ & $\begin{array}{l}\text { Belgium } \\
\text { Canada }\end{array}$ & 86 \\
\hline & & Madhi et $\mathrm{al}^{30}$ & $2010-2011$ & South Africa & 417 \\
\hline & & Heyderman et $\mathrm{al}^{31}$ & $2011-2012$ & $\begin{array}{l}\text { Malawi } \\
\text { South Africa }\end{array}$ & 270 \\
\hline & \multirow[t]{3}{*}{ Pneumococcal } & Binks et al ${ }^{32}$ & $2006-2011$ & Australia & 227 \\
\hline & & Daly et al ${ }^{33}$ & $2000-2003$ & United States & 153 \\
\hline & & $\begin{array}{l}\text { Lopes et al } \\
34\end{array}$ & 2005-2006 & Brazil & 139 \\
\hline & \multirow[t]{2}{*}{ Tdap } & Hoang et al ${ }^{35}$ & $2012-2014$ & Vietnam & 103 \\
\hline & & Villarreal Perez et al ${ }^{36}$ & $2011-2014$ & Mexico & 204 \\
\hline & & Munoz et $\mathrm{al}^{37}$ & $2009-2012$ & United States & 80 \\
\hline
\end{tabular}

Abbreviations: GBS, group B Streptococcus; Hib, Haemophilus influenzae type b; RSV, respiratory syncytial virus; Tdap, tetanus, diphtheria, pertussis. ${ }^{a}$ Ongoing trial.

${ }^{\mathrm{b}}$ All trials after 2000 were included for contact with PI's.

Table 3 Additional trials contact PI's

\begin{tabular}{|c|c|c|c|c|c|}
\hline Pathogen & $\mathrm{NCT}$ & Year & Country & Study size & Phase \\
\hline \multirow[t]{5}{*}{ Influenza } & NCT0099271938 & $2009-2011$ & United States & 84 & II \\
\hline & NCT0117321139 & $2010-2011$ & United States & 183 & II \\
\hline & NCT0090512540 & $2009-2010$ & United States & 102 & II \\
\hline & NCT01577316 41 & $2012-2013$ & Mexico & 240 & $\| / I I I$ \\
\hline & NCT01527825 & $2012-2014$ & South Africa & 800 & III \\
\hline GBS & NCT02046148 ${ }^{43}$ & $2014-2016$ & United States & 75 & $\|$ \\
\hline \multirow[t]{2}{*}{ Pneumococcal } & NCT02628886 & $2016-2019^{a}$ & Gambia & 600 & III \\
\hline & NCT02717494 45 & $2016-2019^{a}$ & Brazil & 345 & II \\
\hline \multirow[t]{2}{*}{ RSV } & NCT02624947 & $2015-2020^{\mathrm{a}}$ & United States & 8,618 & III \\
\hline & NCT02247726 47 & $2014-2016$ & United States & 50 & 11 \\
\hline Pertussis & NCT0055322848 & $2007-2016$ & Canada & 440 & $\| I / I I I$ \\
\hline Tdap & NCT02301702 49 & $2016-2018^{a}$ & Guatemala & 376 & II \\
\hline HIV & NCT00000751 50 & $2001-2007$ & United States & 1,600 & III \\
\hline
\end{tabular}

Abbreviations: GBS, group B Streptococcus; HIV, human immunodeficiency virus; PI, principal investigator; RSV, respiratory syncytial virus; Tdap, tetanus, diphtheria, pertussis.

${ }^{\mathrm{a}}$ Ongoing trial. 
study is the small group of maternal vaccine trials. Unfortunately, 12/30 investigators did not respond to our questionnaire. There may be selection bias. PI's who were aware of PTA and made provisions for PTA may have been more likely to respond to our questionnaire than PI's who did not make them. PI's with PTA provisions in place could be more likely to respond to our questionnaire, and therefore, the proportion of PTA provisions may be an overestimation of the actual provisions implemented.

In conclusion, the publication of international ethical guidelines in 2000 has not resulted in increased publication of ethical provisions in maternal vaccine trial literature. PTA provisions were described in trial protocols, but often the only PTA provision described was publication of the article to make knowledge available instead of providing continued access to interventions that have been proven significant benefit. Future studies should include PTA in their trial protocols, which will increase transparency on the form and content of these provisions. In theory, it can be stated that trials adhere to ethical guidelines and have PTA provisions in place, but in reality, studies do not incorporate all important aspects of PTA provisions into trial planning.

\section{Conflict of Interest}

None declared.

\section{Acknowledgment}

We acknowledge Sharon Nachman (maternal child HIV network, IMPAACT) for providing us data.

\section{References}

1 Marchant A, Sadarangani M, Garand M, et al. Maternal immunisation: collaborating with mother nature. Lancet Infect Dis 2017;17 (07):e197-e208

2 Chu HY, Englund JA. Maternal immunization. Clin Infect Dis 2014; 59(04):560-568

3 Vojtek I, Dieussaert I, Doherty TM, et al. Maternal immunization: where are we now and how to move forward? Ann Med 2018;50 (03):193-208

4 MacDougall DM, Halperin SA. Improving rates of maternal immunization: Challenges and opportunities. Hum Vaccin Immunother 2016;12(04):857-865

5 PATH. RSV Vaccine and mAb Snapshot - PATH Vaccine Resource Library. Cited May 12, 2018. Available at:http://www.path.org/ vaccineresources/details.php?i=1562. Accessed March 29, 2019

6 Council for International Organization of Medical Sciences (CIOMS) in collaboration with the World Health Organization (WHO). Final CIOMS Guidelines 2016. Cited December 16, 2016. Available at: https://cioms.ch/wp-content/uploads/2017/01/WEB-CIOMSEthicalGuidelines.pdf

7 Ethical and Policy Issues in International Research: Clinical Trials in Developing Countries VOLUMEI Report and Recommendations of the National Bioethics Advisory Commission. 2001. Available at: https://bioethicsarchive.georgetown.edu/nbac/clinical/Vol1.pdf

8 The ethics of research related to healthcare in developing countries NUFFIELD COUNCIL ON BIOETHICS. Available at: http:// nuffieldbioethics.org/wp-content/uploads/2014/07/Ethics-ofresearch-related-to-healthcare-in-developing-countries-I.pdf

9 WMA Declaration of Helsinki - Ethical principles for medical research involving human subjects. Available at: https://www. wma.net/policies-post/wma-declaration-of-helsinki-ethical- principles-for-medical-research-involving-human-subjects/. Accessed May 30, 2019

10 Haire B, Jordens C. Mind the gap: an empirical study of post-trial access in HIV biomedical prevention trials. Developing World Bioeth 2015;15(02):85-97

11 Ouzzani M, Hammady H, Fedorowicz Z, Elmagarmid A. Rayyan-a web and mobile app for systematic reviews. Syst Rev 2016;5(01): 210

12 Website World Health Organization. Health topics, vaccines. Available at: http://www.who.int/topics/vaccines/en/. Accessed January 10, 2018

13 Moher D, Liberati A, Tetzlaff J, Altman DG; PRISMA Group. Preferred reporting items for systematic reviews and metaanalyses: the PRISMA statement. PLoS Med 2009;6(07):e1000097

14 Quiambao BP, Nohynek HM, Käyhty H, et al. Immunogenicity and reactogenicity of 23-valent pneumococcal polysaccharide vaccine among pregnant Filipino women and placental transfer of antibodies. Vaccine 2007;25(22):4470-4477

15 Munoz FM, Englund JA, Cheesman CC, et al. Maternal immunization with pneumococcal polysaccharide vaccine in the third trimester of gestation. Vaccine $2001 ; 20(5-6): 826-837$

16 Shahid NS, Steinhoff MC, Roy E, Begum T, Thompson CM, Siber GR. Placental and breast transfer of antibodies after maternal immunization with polysaccharide meningococcal vaccine: a randomized, controlled evaluation. Vaccine 2002;20(17-18):2404-2409

17 Mulholland K, Suara RO, Siber G, et al. Maternal immunization with Haemophilus influenzae type $b$ polysaccharide-tetanus protein conjugate vaccine in The Gambia. JAMA 1996;275(15): 1182-1188

18 Newell KW, Dueñas Lehmann A, LeBlanc DR, Garces Osorio N. The use of toxoid for the prevention of tetanus neonatorum. Final report of a double-blind controlled field trial. Bull World Health Organ 1966;35(06):863-871

19 Koren G, Money D, Boucher M, et al. Serum concentrations, efficacy, and safety of a new, intravenously administered varicella zoster immune globulin in pregnant women. J Clin Pharmacol 2002;42(03):267-274

20 Munoz FM, Piedra PA, Glezen WP. Safety and immunogenicity of respiratory syncytial virus purified fusion protein-2 vaccine in pregnant women. Vaccine 2003;21(24):3465-3467

21 Jackson LA, Patel SM, Swamy GK, et al. Immunogenicity of an inactivated monovalent $2009 \mathrm{H} 1 \mathrm{~N} 1$ influenza vaccine in pregnant women. J Infect Dis 2011;204(06):854-863

22 Tielsch JM, Steinhoff M, Katz J, et al. Designs of two randomized, community-based trials to assess the impact of influenza immunization during pregnancy on respiratory illness among pregnant women and their infants and reproductive outcomes in rural Nepal. BMC Pregnancy Childbirth 2015;15(01):40

23 Omer SB, Richards JL, Madhi SA, et al; BMGF Supported Maternal Influenza Immunization Trials Investigators Group. Three randomized trials of maternal influenza immunization in Mali, Nepal, and South Africa: methods and expectations. Vaccine 2015;33 (32):3801-3812

24 Tsatsaris V, Capitant C, Schmitz T, et al. Maternal immune response and neonatal seroprotection from a single dose of a monovalent nonadjuvanted 2009 Influenza $A(H 1 N 1)$ vaccine: $A$ Single-Group Trial. Ann Intern Med 2011;155:733-741

25 Abzug MJ, Nachman SA, Muresan P, et al. Safety and immunogenicity of 2009 pH1N1 vaccination in HIV-infected pregnant women. Clin Infect Dis 2013;56(10):1488-1497

26 Madhi SA, Cutland CL, Kuwanda L, et al; Maternal Flu Trial (Matflu) Team. Influenza vaccination of pregnant women and protection of their infants. N Engl J Med 2014;371:918-931

27 Zaman K, Roy E, Arifeen SE, et al. Effectiveness of maternal influenza immunization in mothers and infants. N Engl J Med 2008;359(15):1555-1564

28 Salama MM, Hady OA, Ashour W, et al. A randomized controlled trial [corrected] administration of tetanus toxoid (TT) versus 
tetanus and reduced diphtheria (Td) in pregnant women. J Clin Immunol 2009;29(04):524-531

29 Donders GG, Halperin SA, Devlieger R, et al. Maternal immunization with an investigational trivalent group B streptococcal vaccine: a randomized controlled trial. Obstet Gynecol 2016; 127(02):213-221

30 Madhi SA, Cutland CL, Jose L, et al. Safety and immunogenicity of an investigational maternal trivalent group B Streptococcus vaccine in healthy women and their infants: a randomised phase $1 \mathrm{~b} /$ 2 trial. Lancet Infect Dis 2016;16(08):923-934

31 Heyderman RS, Madhi SA, French N, et al. Group B Streptococcus vaccination in pregnant women with or without HIV in Africa: a non-randomised phase 2, open-label, multicentre trial. Lancet Infect Dis 2016;16(05):546-555

32 Binks MJ, Moberley SA, Balloch A, et al. PneuMum: Impact from a randomised controlled trial of maternal 23-valent pneumococcal polysaccharide vaccination on middle ear disease amongst indigenous infants, Northern Territory, Australia. Vaccine 2015;33 (48):6579-6587

33 Daly KA, Scott Giebink G, Lindgren BR, et al. Maternal immunization with pneumococcal 9-valent conjugate vaccine and early infant otitis media. Vaccine 2014;32(51):6948-6955

34 Lopes CR, Berezin EN, Ching TH, Canuto Jde S, Costa VO, Klering ÉM. Ineffectiveness for infants of immunization of mothers with pneumococcal capsular polysaccharide vaccine during pregnancy. Braz J Infect Dis 2009;13(02):104-106

35 Hoang HT, Leuridan E, Maertens K, et al. Pertussis vaccination during pregnancy in Vietnam: Results of a randomized controlled trial Pertussis vaccination during pregnancy. Vaccine 2016;34 (01):151-159

36 Villarreal Pérez JZ, Ramírez Aranda JM, de la O Cavazos M, et al. Randomized clinical trial of the safety and immunogenicity of the Tdap vaccine in pregnant Mexican women. Hum Vaccin Immunother 2017;13(01):128-135

37 Munoz FM, Bond NH, Maccato M, et al. Safety and immunogenicity of tetanus diphtheria and acellular pertussis (Tdap) immunization during pregnancy in mothers and infants: a randomized clinical trial. JAMA 2014;311(17):1760-1769

38 National Institute for Allergy and Infectious Diseases. Novartis H1N1 vaccine in pregnant women. Study NCT00992719. Available at: http://clinicaltrials.gov/ct2/show/results/NCT00992719. Accessed January 12, 2018

39 National Institute for Allergy and Infectious Diseases. 2010-2011 Trivalent Influenza Vaccine (TIV) in Pregnant Women Available at: http://clinicaltrials.gov/ct2/show/NCT01173211?term=influenza\% 2C+infants\&rank=168. Accessed January 12, 2018
40 National Institute for Allergy and Infectious Diseases. A randomized, double-blind trial on the safety and immunogenicity of inactivated 2008/2009 trivalent influenza vaccine in pregnant women. Available at: http://clinicaltrials.gov/ct2/show/ NCT01173211?term=influenza\%2C + infants\&rank=168. Accessed January 12, 2018

41 Garcia LG. Clinical trial to evaluate the immunogenicity and safety of the 2011-2012 vaccine against seasonal influenza on pregnant women. Available at: http://clinicaltrials.gov/ct2/show/record/ NCT01577316. Accessed January 12, 2018

42 Groome M. Immunogenicity and Safety of Different Dosing Schedules of Trivalent Influenza Vaccine in HIV-infected Pregnant Women. Available at: https://clinicaltrials.gov/ct2/show/ NCT01527825. Accessed January 12, 2018

43 GlaxoSmithKline, Safety and Immunogenicity of a Trivalent Group B Streptococcus Vaccine in Healthy Pregnant Women. Available at: https://clinicaltrials.gov/ct2/show/NCT02046148. Accessed January 22, 2018

44 Medical Research Council Unit. The Gambia. Protecting from Pneumococcus in Early Life (The PROPEL Trial) (PROPEL). Available at: https://clinicaltrials.gov/ct2/show/NCT02628886. Accessed January 22, 2018

45 Westat. Safety and Immunogenicity of Anti-Pneumococcal Vaccines in HIV-infected Pregnant Women. Available at: https:// clinicaltrials.gov/ct2/show/NCT02717494. Accessed January 22, 2018

46 Novavax. A Study to Determine the Safety and Efficacy of the RSV F Vaccine to Protect Infants Via Maternal Immunization. Available at: https://clinicaltrials.gov/ct2/show/NCT02624947. Accessed January 22, 2018

47 Novavax. RSV F Vaccine Maternal Immunization Study in Healthy Third-trimester Pregnant Women. Available at: https://clinicaltrials.gov/ct2/show/NCT02247726. Accessed January 22, 2018

48 Halperin S. Pertussis Maternal Immunization Study. Available at: https://www.clinicaltrials.gov/ct2/show/NCT00553228. Accessed January 22, 2018

49 Omer S. Maternal Tdap Immunization in Guatemala. Available at: https://clinicaltrials.gov/ct2/show/NCT02301702. Accessed January 22,2018

50 National Institute of Allergy and Infectious Diseases (NIAID). A Phase III Randomized, Double-Blind, Controlled Study of the Use of Anti-HIV Immune Serum Globulin (HIVIG) for the Prevention of Maternal-Fetal HIV Transmission in Pregnant Women and Newborns Receiving Zidovudine (AZT). Available at: https:// clinicaltrials.gov/ct2/show/study/NCT00000751?show_locs=Y\# locn. Accessed January 22, 2018 